\title{
Cardiac Anatomy
}

\author{
Augusta Pelosi ${ }^{1}$ and Jack Rubinstein ${ }^{2}$ \\ ${ }^{1}$ Michigan State University, \\ ${ }^{2}$ University of Cincinnati \\ USA
}

\section{Introduction}

The understanding of development and formation of normal anatomic structures is fundamental to comprehend electrocardiograms, conduction patterns and abnormalities. The aim of this chapter is to provide an overview of the cardiac chambers, the valves, the cardiac vasculature and the relation with the electrical conduction. The chapter will also review embryologic features of the cardiac structures.

\section{Embryology}

The heart develops in several sequential steps. The order and the completion of the entire process during fetal life are fundamental for having a post-birth functional and normally structured heart and conduction system. This section will review the basic steps of this process through the development of the cardiac chambers, the septa formation, the development of the major vessels, and the circulation before and after birth.

The heart is the first internal organ to form and become functional in the vertebrate development, (Srivastava, 2000) starting the first beats in humans by day 22 and the circulation by day 27-29. (Kelly, 2002, Pensky, 1992) Mesodermal cells migrate to an anterior and lateral position where they form bilateral primary heart fields (DeHaan 1963) which then coalesce to form two lateral endocardial tubes. (Harvey, 1999; Covin, 1998) The tubes fuse and merge into one endocardial tube surrounded by splanchnopleuric-derived myocardium. (Covin 1998) The cephalic and lateral folding of the embryo push the endocardial tube from a lateral position into the ventral midline. (Sherman, 2001) During the first month of gestation, the primitive, straight cardiac tube starts developing defined spaces with constrictions in series which will become the future cardiac structures: the sinu-atrium (most caudal), the primitive ventricle, the bulbus cordis, and the truncus arteriosus (most cephalad). (Abdulla, 2004; Angelini,1995) The primitive ventricle will eventually become the left ventricle whereas the right ventricle will develop from the proximal portion of the bulbus cordis. The distal portion of the right ventricle will form the outflow of both ventricles and the truncus arteriosus will form the root of the aorta and pulmonary arteries. (Abdulla, 2004) The linear heart tube becomes polarized with a posterior inflow pole (venous pole) and an anterior outflow pole (arterial). The truncus arteriosus is connected to the aortic sac and through the aortic arches to the dorsal aorta. (Pensky, 1992) Conversely, the sinuatrium, composted of the primitive atrium and the sinus venosus, receives the vitelline veins (from the yolk sac, also draining the gastrointestinal system and the portal circulation), the 
common cardinal veins (draining the anterior cardinal vein coming from the anterior part of the embryo), the posterior cardinal vein (from the posterior part of the embryo), and the umbilical veins (from the primitive placenta).

Between day 22 and 28, the heart begins to fold and loop, as the epicardial cells start covering the outside layer of the heart tube. (Sherman, 2001) The heart tube loops because of intrinsic properties of the myocardium which encode for the initiation of the looping process, rather than due to asynchronous growth compared to the outside structures. (VanMierop, 1979) This process occurs prior to the formation of the chambers within the heart tube. By day 28, the atria move in a position higher than the ventricles, with the outside marks which refer to the sinus venous, common atrial chamber, atrioventricular sulcus, ventricular chamber, and conotruncus (outflow tracts). (Sherman, 2001) The bulboventricular sulcus, corresponding to the inner bulboventricular fold, starts to become visible from the outside. The heart assumes a U-shape where the bulbus cordis is located to the right and the primitive ventricle forms the left arm. The paired sinus venosus gives rise to the sinus horns. The two sinus horns are paired structures, which then fuse to form a transverse sinus venosus. (Abdulla, 2004) The entrance of the sinus venosus shifts rightward to enter into the right atrium. The right AV canal and right ventricle expand and align so that atria and ventricles are over each other, determining the alignment of the simultaneous left atrium and ventricle, and the proper alignment of the future aorta. (Sherman, 2001) The common atrioventricular junction changes into the atrioventricular canal, connecting the left side of the common atrium to the primitive ventricle. (Pensky, 1992) The inner surface is smooth except for the trabeculations, present at the level of the bulboventricular foramen. As the atrium grows, it pushes the bulbus cordis in the space between the two atria. (VanMierop, 1979) The symmetry in the development is lost by weeks $4-8$ in the cardiac chambers and the aortic arches. (Kirby 2002) The cardiac neural crest, originating from the neural tube in the region of the three somites, starts migrating through the aortic arches 3, 4, and 6 into the developing outflow tract (week 5 and 6). These cells are responsible for septation of the outflow tract and ventricles, the anterior parasympathetic plexus, (Sherman, 2001) the leaflets of the atrioventricular valves, and the cardiac conduction system. (Hildreth, 2008; Poelman,1999)

\subsection{Cardiac chambers and septation}

Atria. The auricles of the right and left atria originate from the primitive atria, while the smooth sections come from the tissue originating from the venous blood vessels (sinus venosus on the right and pulmonary veins on the left). At day 35 an indentation provoked by the bulbus cordis and truncus arteriosus begins to create, on the inner surface of the common atrium, a wedge of tissue called septum primum, which extends into the common atrium separating it into a left and right compartment. (Steding, 1994) The septum primum allows a concave-shaped edge to form permitting shunting of blood from right to left. Apoptosis of cells in the superior edge of the septum primum forms a new foramen called the ostium secundum. (VanMierop, 1979) The endocardial cushions fuse with the ostium primum obliterating it. The septum secundum forms to the right of the septum primum. The septum is incomplete with a foramen ovale near the floor of the right atrium allowing passage of blood from right to left through the foramen ovale. (Abdulla, 2004; Angelini, 1995) Both septum primum and secundum fuse with the septum intermedium of the AV cushion.

Ventricles. The primary muscular ventricular septum begins to grow during the fifth week from the apex toward the atrioventricular valves. The initial growth is due to the growth of 
the two ventricles in the opposite direction. (Abdulla, 2004) The trabeculations from the inlet regions cause the formation of a septum which grows with a mildly different angle. This septum will meet the primary septum and provoke the primary septum to protrude into the right ventricular cavity forming the trabeculae septomarginalis. The high portion of the interventricular septum has a concave upper ridge which forms the interventricular foramen. The foramen closes at the end of week 7 by the posterior endocardial tissue, and the right and left bulbar ridges. (Abdulla, 2004) The majoriy of the muscular part of the septum is formed by the fusion of these septa. The outflow tract septum has grown down on the upper ridge of the muscular ventricular septum and onto the inferior endocardial cushion, separating the ventricular chambers.

\subsection{Great vessels and arterial and venous development}

Outlow tract septation. The mechanism of outflow septation is somewhat controversial. The proximal portion of the outflow tract septum septates by the fusion of the endocardial cushions and joints the atrioventricular endocardial cushion tissue and the ventricular septum. (Waldo 1998) The distal portion septates by intervention of the cardiac neural crest. (Kirby 2002). The septation of the outflow (conotruncus) is coordinated with the septation of the ventricles and atria. The septa fuse with the atrioventricular (AV) cushions dividing the left and right AV canals. Several theories for this process have been proposed. In general, three embryologic areas can be considered: the conus, the truncus and the aortopulmonary. (Abdulla, 2004) Each develops a ridge which is responsible for the formation of the septum between the fourth (future aortic arch) and the sixth (future pulmonary artery) arches. The truncus ridges form the septum between the ascending aorta and the main pulmonary artery, whereas the conus ridge forms the septation between the right and left outflow tract. (Abdulla, 2004)

Pulmonary arteries and veins. The main pulmonary artery develops from the trunctus arteriosus. The distal main and the right pulmonary artery develop from the ventral sixth aortic arch artery. The distal right and left have a different origin, deriving from the post branchial arteries. The ductus arteriosus develops from the left sixth aortic arch artery. The pulmonary venous system originates at the level of the left atrium, from a primitive vein sprout. These vessels anastomose with the veins extending from the bronchial bud. (Abdulla, 2004)

Systemic veins. The sinus venosus initially communicates with the common atrium, by week 7 the axis moves toward the right creating a connection between the right atrium and the sinus venosus. Around weeks 7-8 several changes occur to the venous system. The cardinal system is modified because the proximal left cardinal vein anastomoses with the right anterior cardinal vein via the left brachiocephalic vein creating the superior vena cava. The intermediate portion of the left cardinal vein degenerates and the portion close to the heart becomes the coronary sinus. (James, 2001) The left posterior cardinal vein degenerates, the right posterior cardinal vein becomes the azygous vein, and the left sinus horn contributes to the coronary sinus. The vitelline veins also undergo several changes: the right vitelline vein becomes the inferior vena cava. The course of the umbilical veins (coming from the placenta) is also modified by the degeneration of the left umbilical vein while the right umbilical vein connects to the right vitelline vein through the ductus venosus (derived from the vitelline veins). The veins draining into the left sinus venosus (left cardianal, umbilical, and vitelline) degenerate and the left sinus venosus becomes the coronary sinus, draining only the venous circulation of the heart. (Abdulla, 2004) 
Aortic arches. The dorsal and ventral aorta are connected by six paired aortic arches. The first pair of aortic arches contributes to form the external carotid arteries. The second pairs regresses except a small portion forming the hyoid and stapedial arteries. The third pair forms the common and proximal part of the internal carotid arteries (the distal part is formed by the dorsal aorta). The left fourth arch forms the aortic arch maintaining the connection between the ventral to the dorsal aorta. The right fourth constitutes part of the right subclavian. The fifth pair regresses. The sixth evolves into the main and right pulmonary artery, whereas the distal portion forms the ductus arteriosus. (Abdulla, 2004)

Coronary arteries. The proepicardial organ surrounds the myocardium as the heart starts looping, forming the epicardium. (Komiyama, 1996) These cells form the coronary vasculature. These cells originate from an independent population of splanchnopleuric mesoderm cells and migrate into the primary heart tube. The coronary arteries (smooth muscle, endothelial, and connective tissue) form prior to migration into the heart tube. (Harvey, 1999; Mikawa, 1996).

\subsection{Atrioventricular canal}

The atrioventricular valves form during the 5th to 8th weeks. (Larsen, 1997) By the end of the $5^{\text {th }}$ week, parts of the ventricles are visible and the left ventricle supports most of the circumference of the AV canal. The endocardial cushion starts from the sides of the atrioventricular junction with a superior and inferior cushion. They move toward the center of the canal forming the septum intermedium and the right and left atrioventricular orifices. (Snell, 2008) The cushion is also responsible for completing the closure of the interatrial communication at the level of the septum primum. (Van Mierop, 1979) Migration of the AV canal to the right and the ventricular septum to the left serves to align each ventricle with its appropriate AV valve. The formation of the valves starts with an asynchronous growth of the atria in comparison to the atrioventricular junction. The sulcus invaginates into the ventricular cavity with the formation of a hanging flap which is covered by the atrial and the ventricular tissue. (Abdulla, 2004)

\subsection{Conduction system}

The primary myocardium originates the contracting and the conducting tissue. The origin of the sinus and atrioventricular (AV) node is not well known. The cells seem to originate at the original connection of the sinus venosus with the right and left superior cardinal veins. These small groups of cells follow the cardinal veins as they move to their final destination. The right cardinal vein becomes the superior vena cava and maintains its connection to the sinus (SA) node. The left cardinal vein becomes the superior left vena cava and it is transformed into the coronary sinus, leaving sometimes a small vessel (the vein of Marshall). In general, the conducting system is formed by the accumulation of conduction myocardial tissue around the bulboventricular foramen. The dorsal portion becomes the bundle of His, whereas the lower tracts form the left and right bundle branches. Portions of this specialized tissue (right atrioventricular ring and the retroaortic branch) form but then disappear during normal development. (Abdulla, 2004)

\subsection{Circulation}

The fetal circulation is in parallel and dependent on the placenta because the lungs are not functional. The circulation in the adult becomes in series. There are several differences 
between the two systems. The oxygenated blood, through the umbilical veins, reaches the heart of the fetus and flows away once deoxygenated, through the umbilical arteries. Once entered the fetal body, the blood bypasses the liver passing through the ductus venosus and enters the inferior vena cava to reach the right atrium. The position of the vena cava and the ligament of the inferior vena cava allow the blood to flow into the foramen ovale and into the left atrium. The foramen ovale appears like a valve formed by the foramen ovale and the ostium secundum. Part of the oxygenated blood in the right atrium mixes with the deoxygenated blood returning from the systemic circulation. From the left atrium, the blood follows the normal circulation and reaches the left ventricle, where it is pushed by the ventricular systole into the aorta. The venous return to the right atrium via superior vena cava follows the blood flow through the tricuspid valve into the pulmonary artery. Once the blood reaches the main pulmonary artery, it is diverted by the high pulmonary resistance into the ductus arteriosus at the level of the aortic isthmus. Only one-tenth of the right ventricular output reaches the lungs. The blood follows the descending aorta and returns to the placenta via the umbilical arteries. By the third month, the heart and major vessels are formed. However, the transition to the adult circulation occurs shortly after birth, when the umbilical cord is cut and the neonate takes the first breath. The lung expansion produces a drop in pulmonary resistance and increase in pressure inside the left atrium. Therefore, the pressure in the left atrium becomes mildly higher than the pressure on the right atrium, determining a closure of the valve flap associated with the foramen ovale, which transforms into a visible depression in the interatrial septum, called fossa ovalis. The increased concentration of prostaglandins, occurring with the parturition, results in the closure of the ductus arteriosus, which transforms into the ligamentum arteriosum. (Friedman, 1993)

The dramatic changes occurring with birth determine rapid transition toward the adult circulation with complete separation of the left and right compartments. The heart is functionally and anatomically divided into left and right. Each side has two chambers: atrium and ventricle, one major artery per side (aorta to the left and pulmonary artery to the right), and a venous return system (venae cavae to the right and pulmonary veins to the left). The deoxygenated blood returns to the right atrium from the systemic circulation through the venae cavae, and flows into the right ventricle through the tricuspid valve; it is then pushed into the lungs through the pulmonary valve and artery. The blood, now oxygenated, returns to the left atrium via the pulmonary veins, goes into the left ventricle through the mitral valve, and it is pushed to the rest of the body via the aorta.

\section{Cardiac anatomy and thoracic cavity}

The thoracic cavity can be divided into several compartments by imaginary lines. The mediastinum is divided into superior and inferior mediastinum by the transverse thoracic plane, which extends from the sternal angle to the space between the thoracic vertebrae T4 and T5. This line divides the thoracic cavity into superior and inferior mediastinum. The inferior mediastinum can be divided into an anterior, middle and posterior mediastinum. (Snell, 2008)

The anterior mediastium is bounded by a line crossing the thorax from the trachea to the xiphoid, just anterior to the pericardium. The middle mediastinum is the central part and contains the heart and the pericardium. The posterior mediastinum is contained between the pericardium anteriorly and the anterior surfaces of the bodies of the thoracic vertebrae (T5-T12). (Snell, 2008) Superiorly the thorax narrows as it enters the neck (1 ${ }^{\text {st }}$ ribs, the 
manubrium and the $1^{\text {st }}$ thoracic vertebra), and inferiorly the anatomic separation with the abdomen is well defined by the diaphragm. Along the midline, the mediastinum is responsible for the separation into two equal cavities, the left and the right pulmonary cavities.

The thoracic wall is formed of 12 ribs, the thoracic vertebrae, interventional discs, and the sternum. The ribs articulate with the thoracic vertebrae. The first 7 ribs are described as "true" because they articulate directly or indirectly with the sternum. The following ribs (810) are referred as "false" because they connect indirectly to the sternum. Ribs 11 and 12 are referred as "floating" ribs because they do not connect to the sternum. A posterior depression to the rib accommodates the intercostal neurovascular bundles, located between the internal and innermost intercostals layers. The sternum, formed by sternebrae, is a flat bone composed of three parts: the manubrium, body, and the xiphoid process. The muscles of the thoracic cavity play a fundamental role in respiration and movement of the thoracic cavity. The intercostal muscles are composed of three layers: the external, internal and innermost intercostals muscles. The diaphragm attaches to the upper lumbar vertebrae at the level of the right and left crura (lumbar vertebra 1 through 3). Laterally the diaphragm attaches to the abdominal wall musculature and to the xiphoid process. The diaphragmatic dome is formed by a muscular external portion and a central aponeurosis. It contributes to respiration by contracting during respiration. The central tendon contains the opening of the inferior vena cava. In the right crus the esophagus passes through the diaphragm, while the aorta passes from the thorax behind the diaphragm. The transit of these structures occurs at the level of the vertebrae 8, 10 and 12. (Netter, 2010)

The thoracic cavity contains the heart, lungs, great vessels, esophagus, trachea, thoracic duct, thymus and the autonomic innervations. The pleura covers the entire thoracic cavity.

The aortic arch moves from right to left as it enters the posterior mediastinum and becomes vertical as it crosses T4. Through the posterior mediastinum it moves to the middle at the level of T5. It crosses the diaphragm via the aortic hiatus and enters the abdomen at the level of T12. It gives off the posterior intercostal arteries and the subcostal artery, the bronchial and the esophageal branches. At the level of the aortic arch, three arteries branch off: the most anterior is the brachiocephalic artery, the left common carotid artery, and the left subclavian artery. The brachiocephalic artery bifurcates to become the right common carotid and the right subclavian arteries. The subclavian arteries form the axillary and brachial arteries. The subclavian artery gives off the internal thoracic arteries which reenter the superior mediastinum along the sternum. Occasionally there is an additional artery from the aortic arch. (Netter, 2010)

The internal jugular vein and the subclavian vein converge to form the brachiocephalic (or innominate) veins. These veins form two large trunks in either sides of the root of the neck and penetrate the superior mediastinum where they receive the contribution of the internal thoracic, inferior thyroid veins and the small pericardiophrenic veins, and the superior intercostal veins. The left crosses obliquely to join the right and form the superior vena cava. The superior vena cava enters the pericardial sac in the middle mediastinum to reach the right atrium from a superior position. The inferior vena cava enters from below. The azygous system consists of the azygous vein on the right and the hemiazygous and accessory hemiazygous vein on the left. The azygous and hemiazygous receives the blood from the abdomen and the subcostal vein. The azygous begins in the abdomen and enters the thorax via the aortic hiatus. It curves over the lung and drains into the superior vena cava. The hemiazygous crosses the diaphragm through the left crus and remains posterior 
the aorta, esophagus and thoracic duct before terminating into the azygous vein. The accessory hemiazygous vein either joins the azygous or terminates in the hemiazygous.

The pulmonary trunk arises from the right ventricle on the anterior surface of the heart directing to the left and posteriorly, passing anteriorly to the base of the aorta. The pulmonary artery bifurcates into the left and right pulmonary artery. The right enters the right lung passing under the aortic arch. The ligamentum arteriosum connects the left pulmonary artery to the aortic arch. (Netter, 2010)

The trachea terminates at the bifurcation into the bronchi at the level of the superior mediastinum. The esophagus descends behind the trachea at the level of the superior mediastinum, entering the abdominal cavity through the diaphragm at the level of T10. (Netter, 2010) The thymus is found in the anterior portion of the superior mediastinum. It is directly behind the manubrium and may extend into the anterior mediastinum It contacts the aorta, the left brachiocephalic vein and the trachea. The aortic arch is located to the left of the trachea and esophagus. The azygous vein crosses anteriorly to them and to the right. The thoracic duct enters into the posterior mediastinum through the aortic hiatus and travels between the thoracic aorta and the azygous vein behind the esophagus. It then drains into the left venous system close to the junction of the internal jugular and subclavian veins.

The superior mediastinum is crossed by the vagus and the phrenic nerve. The phrenic nerves originate from the ventral rami at the cervical levels 3, 4, 5. (Snell, 2008) They run along the neck, entering the thorax under the internal thoracic artery. The right nerve passes through the superior mediastinum, lateral to the right brachiocephalic vein and the superior vena cava. (Aquino, 2001) The left nerve passes lateral to the left subclavian artery and the aortic arch. Both nerves descend along the pericardium crossing through the middle mediastinum with the pericardiacphrenic artery (branch of the internal thoracic artery) and vein which empties into the subclavian vein. (Aquino, 2001) The vagus nerves leave the skull through the jugular foramen and descend along the carotid sheath. They give off cardiac branches in the neck (superior and inferior cardiac nerves) and a low number of small cardiac nerves in the superior mediastinum (thoracic cardiac branches), providing parasympathetic innervation to the heart via the cardiac nerve plexus. (Aquino, 2001; Snell, 2008) The right nerve descends between the lung and the trachea and it gives off the recurrent laryngeal nerve before entering the superior mediastinum (at the level of the right subclavian). (Aquino, 2001) It assists in the formation of the pulmonary plexus and then contributes to the formation of the esophageal plexus. (Snell, 2008) Conversely, the left descends between the carotid artery and the left subclavian artery and passes lateral to the aortic arch where it gives off the left recurrent laryngeal nerve, which passes under the arch just posterior to the ligamentum arteriosum. (Aquino, 2001) The left portion follows laterally the trachea and esophagus and ramifies into the esophageal plexus. (Aquino, 2001) Therefore the esophageal plexus, created by the right and left vagus in the middle mediastinum, forms the anterior and posterior vagal trunk which enters the abdomen through the esophageal hiatus. (Aquino, 2001) The sympathetic innervation is constituted by paired chains extending from the neck to the diaphram. (Aquino, 2001; Netter, 2010) The superior, middle, and inferior cardiac nerves provide postganglionic fibers to the heart providing sympathetic innervation. The thoracic ganglion and the inferior cervical ganglion form the "stellate ganglion" giving off the inferior cardiac nerve. (Snell, 2008) The cardiac plexus is a network of sympathetic and parasympathetic nerves primarily innervating the conduction system and the atria. 


\subsection{Heart in the thoracic cavity and external anatomy}

The heart is located within the thoracic cavity in the middle of the inferior mediastinum, it occupies a large portion of this space. It is surrounded by the pericardium. The pericardium is a mesothelium formed by an external fibrous and an internal serous surface. The external parietal surface is composed of the two layers: an external thickened fibrous on the outside and an inner serous surface on the inside. (Snell, 2008) The two layers are adhered. The internal serous membrane presents a parietal and a visceral layer. The inner visceral layer covers the heart forming the epicardium. There is a potential space between the visceral and parietal layers containing small amount of fluid produced by the mesothelial cells. The parietal pericardium covers the aorta, pulmonary artery forming the arterial reflections and the superior, inferior vena cava and pulmonary veins forming the venous reflections. The oblique pericardial sinus is formed by the venous reflection of the inferior vena cava and pulmonary veins. The transverse pericardial sinus is formed between the arterial reflections and the venous reflections. Inferiorly, the parietal pericardium is attached to the diaphragm. Anteriorly, the superior and inferior sternopericardiac ligaments secure the parietal pericardium to the manubrium and the xiphoid process, respectively. (Netter, 2010)

Within the pericardium, the heart is a muscular four chamber organ connected to the rest of the thoracic cavity by two inflow and two outflow vessels. The orientation of the cardiac axis is oblique resulting in the apex being anterior and toward the left and a base located superior, posterior, and to the right of midline. The heartbeat is easily palpated between the $5^{\text {th }}$ and $6^{\text {th }}$ ribs. The left border is formed by the left ventricle and the right border by the right atrium. The right ventricle is located anteriorly while the left atrium is located posteriorly in front of the spine. The external separation between the left and right ventricle, highlighting the interventricular septum, is the anterior interventricular sulcus (groove), which contains the anterior interventricular descending branch of the left coronary artery and the posterior interventricular sulcus (groove), containing the posterior interventricular (descending) artery and middle cardiac vein. The anatomical separation between the right atrium and right ventricle is provided by the right atrioventricular sulcus (coronary groove) in which the right coronary artery transits. The separation between the left atrium and left ventricle is highlighted by the left atrioventricular sulcus (coronary sulcus) containing the coronary sinus. The plane of this sulcus also contains the cardiac skeleton and the valves. The interatrial septum posteriorly is called the atrial sulcus. The intersection of the atrial sulcus and the posterior interventricular sulcus with the perpendicular coronary sulcus forms a cross shape on the posterior surface, called the crux cordis. (Netter, 2010)

\section{Anatomy of the cardiac chambers, valves, and major vessels}

The cardiac skeleton provides a scaffold for the attachment of the atrial and ventricular myocardium, the four valves and electrically insulates the atria from the ventricle. The fibrous structure present four rings for the opening of the aortic semilunar valve in the center and the other opening attached to it. The center is triangular shaped, called right fibrous trigone or central fibrous body, and it is included among the rings of the aortic semilunar valve, the medial parts of the tricuspid and mitral valve. The smallest left trigone is formed between the aortic semilunar valve and the anterior cusp of the mitral valve. The fibroelastic tissue from the right and left trigone partially encircle the AV opening to form the tricuspid and mitral annulus or annulus fibrosus. (Iaizzo, 2005) The annuli provide attachment to the myocardium and the AV leaflets. Strong collagen tissue from the right and 
left trigone also encircles the semilunar rings. The membranous septum provides support to the medial cusps of the aortic valve and continues superiorly to form the atrial septum. The tendon of Todaro is a fibrous extension of the membranous septum that is continuous with the Eustachian valve of the inferior vena cava. (Netter, 2010)

\subsection{Right atrium and tricuspid valve}

The interior of the right atrium has three distinct parts. The posterior portion of the right atrium has a smooth wall and is referred as the sinus venarum (the embryologic right horn of the sinus venosus). (Netter, 2010) The smooth posterior wall receives the superior and the inferior venae cavae and the coronary sinus. The anterior portion is very thin-walled but along its walls run the muscle bundles called pectinate muscles. (Snell, 2008) The physical separation between the anterior and posterior parts is a ridge of muscle, the crista terminalis. (Snell, 2008) In the embryo, the crista terminalis separates the sinus venosus and the primitive atrium. (Abdulla, 2004) This prominence corresponds to the external sulcus terminalis. (Snell, 2008) It is more prominent on the side of the superior venae cava and then fades out toward the inferior vena cava. The pectinate muscles continue into the right auricle, a triangular-shaped space on the superior portion of the right atrium. (Snell, 2008) The right auricle is broad and blunt. It extends from the superior vena cava almost to the inferior vena cava. (Netter, 2010) The inferior border of the right atrium contains the ostium of the vena cava and the ostium of the coronary sinus. The ostium of the vena cava opens anteriorly with a fold of tissue, the inferior vena cava Eustachian valve (fetal remnant). It is sometimes absent, but when present, it may appear with several openings, called network of Chiari. The coronary sinus opening is located anteriorly and inferiorly to the orifice of the inferior vena cava. It is sometimes guarded by a valve-like structure, called the coronarysinus Thebesian valve. These two venous valves insert into a prominent ridge, the Eustachian ridge (sinus septum) which runs medial-lateral across the inferior border of the atrium and separates the os of the coronary sinus and inferior vena cava. Both valves originate from a large embryonic right venous valve. The interatrial septum forms the posteromedial wall of the right atrium. The interatrial septum has an interatrial and an atrioventricular part. It originates from the embryologic septum primum and septum secundum. It is muscular except for a central fibrous depression, called fossa ovalis resulting from the foramen ovale. It is surrounded by the limbus fossae ovalis, a muscular ridge surrounding the depression. The fossa ovalis is positioned anterior and superior to the ostia of both the inferior vena cava and the coronary sinus. A tendinous structure, the tendon of Todaro, connects the valve of the inferior vena cava to the central fibrous body of the cardiac skeleton. It appears as a fibrous extension of the membranous portion of the interventricular septum. It moves obliquely within the Eustachian ridge and separates the fossa ovalis from the coronary sinus below. This tendon has a structural role to support the inferior vena cava and is a useful landmark to approximate the location of the AV node.The conduction system is also closely associated with the right atrium. The SA node is located between the myocardium and the epicardium in the superior portion of the right atrium. To localize the SA node, the intersection of the line passing through the sulcus terminalis, the lateral border of the superior vena cava and the superior border of the right auricle, identify the position of the SA node. To approximate the location of the AV node, it is necessary to identify the triangle of Koch: the base passes through the coronary sinus; the sides are the septal leaflets of the tricuspid valve and the tendon of Todaro. 
The tricuspid valve annulus lies on the floor of the right atrium, attached to the membranous portion of the septum. The tricuspid valve apparatus and the atrioventricular valve, is formed by an annulus, leaflets, papillary muscles, and the chordae tendinae. The $\mathrm{AV}$ orifice is reinforced by the annulus fibrosus of the cardiac skeleton. The three leaflets are the anterior (superior), posterior (inferior), and medial (septal). The leaflets have a smooth surface on the atrial side presenting only small nodules from the edges, called the noduli albini. (Netter, 2010) These appear to be present mostly in children and assure complete coaptation of the valve upon closure. The atrial side of the the leaflet is smooth whereas the ventricular surface is more irregular and provides insertion of the chordae. The anterior leaflet of the valve is the largest and extends from the medial border of the ventricular septum to the anterior free wall. The posterior leaflet extends from the lateral free wall to the posterior portion of the ventricular septum. The septal leaflet extends from the annulus to the medial side of the interventricular septum.

The primary order of chordae connects the papillary muscle to the free edge of the leaflets with several fine strands, impeding the valve leaflets from inverting. The secondary order chordae connect the papillary muscle to a ventricular portion of the leaflet. They are stronger and less numerous, providing the major stability to the valve. The tertiary order connects the ventricular myocardium to the leaflet. They form bands which can contain muscles. The commissures connect the leaflets and they are named after the connected leaflets: anteroseptal, anteroposterior and posteroseptal. They never reach the annulus so they provide only incomplete separation of the leaflets. (Netter, 2010)

\subsection{Right ventricle and pulmonic valve}

The right ventricular cavity is separated into two sections: posteroinferior portion containing the inflow with the tricuspid valve, and the anterosuperior outflow portion, containing the pulmonary trunk. The separation between these two portions is formed by a small ridge of several muscular bands, the crista supraventricularis, the septal trabeculae (septal band), and the moderator band. These muscle bundles form the trabeculae septomarginalis, which form a semicircular arch (delineation of the outflow tract). (Netter, 2010) The inflow portion is heavily trabeculated by coarse trabeculae carneae, the outflow portion is named infundibulum and contains only a few trabeculae, and the subpulmonic area has a smooth surface. (Snell, 2008) Several papillary muscles connect the walls to the leaflets via the chordae tendinae. The anterior and the medial papillary muscles are always present, while additional papillary muscles can be present in variable number. The medial papillary muscle is located where the crista supraventricularis meets the septal band. It provides attachment to the chordae tendinae to the posterior and septal leaflet of the tricuspid valve. (Rogers, 2009) It is small in the adult heart. The largest papillary muscle is the anterior papillary muscle, which receives the chordae from the anterior and posterior leaflets of the tricuspid valve (Rogers, 2009) and it is located at the apex of the right ventricle.(Netter, 2010) The other papillary muscles (posterior and septal) are small and attach via chordae to the posterior and medial leaflet.

The outflow portion originates superiorly in the right ventricle. The pulmonary trunk bifurcates into right and left pulmonary arteries. The ligamentum arteriosus, remnant of the fetal ductus arteriosus, connects the bifurcation of the pulmonary artery to the inferior surface of the aortic arch. The pulmonary valve, as the other semilunar valve, differs from the atrioventricular valves. There is not a defined annulus to support the valve. The first portion 
of the vessel expands to form three pouches, the sinus of Valsalva which are mildly developed in the pulmonary artery compared to the aorta. The valvular leaflets are smooth and thin with a small fibrous nodule (nodulus Arantii) at the center of the free edge. (Netter, 2010)

\subsection{Left atrium and mitral valve}

The left atrium has a smooth surface with a transverse axis larger than the vertical and sagittal axes. The internal surface of the left atrial vestibule is smooth because the pectinate muscles are confined within the auricle. The left auricle is a continuation of the upper anterior portion of the left atrium. It is located anteriorly over the atrioventricular sulcus. Its shape is variable but it tends to be narrowed and pointed. (Ho, 2002; Ho, 2009) Its inner surface is irregular by the pectinate muscles. The septal surface is mostly smooth except for the area of the foramen ovale. (Snell, 2008) The left atrium receives two or three pulmonary veins from the right and two pulmonary veins from the left lungs. (Netter, 2010)

The mitral valve apparatus, as the other atrioventricular valve, is formed by an annulus, leaflets, papillary muscles and the chordae tendinae. The annulus is reinforced by the annulus fibrosus of the cardiac skeleton, supporting the posterior and lateral two-thirds of the mitral annulus. At the level of the right and left fibrous trigone, the annulus is reinforced by fibrous tissue. On the medial side, the attachment of the fibrous support of the aortic semilunar valve provides additional support. The valve has two leaflets: the anterior, also called medial or aortic, and the posterior (inferior or mural). (VanMieghen, 2010) The shape of the anterior leaflet resembles a trapezoidal shape. (Netter, 2010) The posterior leaflet is quite narrow and it subdivided into an anterior, central and posterior shape. When the valve closes, there is significant overlap of the leaflets. (Bolling, 2006) The connection between the leaflets is provided by the commissures, anterolateral and posteromedial. They never reach the annulus so they provide only incomplete separation of the leaflets. The leaflets have a smooth surface on the atrial side presenting only small nodules from the edges, called the noduli albini. These appear to be present mostly in children and assure complete coaptation of the valve upon closure. (Netter, 2010) The ventricular surface is more irregular and provides insertion of the chordae. The primary order of chordae connects the papillary muscle to the free edge of the leaflets with several fine strands, impeding the valve leaflets from inverting. The secondary order connects the papillary muscle to a more ventricular portion of the leaflet. They are stronger and less numerous, providing the greatest stability to the valve. The tertiary order connects the ventricular myocardium to the leaflet. (Bolling, 2006; Netter, 2010) They form bands which can contain muscles. The primary and secondary orders are constituted partially by muscle in the mitral apparatus. This feature is indicative of the common embryologic origin of the papillary muscles, the chordae and most of the leaflets from the embryonic ventricular trabeculae, which were muscular in origin. (Netter, 2010)

\subsection{Left ventricle and aortic valve}

The left ventricle has two separate portions, the inflow and the outflow separated by a fibrous band which provides attachment to the anterior mitral leaflet and the left and posterior aortic valve leaflets. The left ventricle is physiologically thicker than the right ventricle. The trabeculae carnae, presents mostly toward the apex, from the wall of the left ventricle but the muscular ridges are finer and less coarse compared to the walls of the right 
ventricle. (Snell, 2008) The wall of the basilar portion is smooth. The interventricular septum is muscular except in the area below the right and posterior aortic leaflets which is membranous. The separation between the muscular and membranous part is called limbus marginalis. (Netter, 2010) The membranous portion is divided into two parts by the origin of the medial leaflet of the tricuspid valve, creating an upper portion, the atrioventricular part (between the left ventricle and the right atrium) and the lower one, the interventricular part (between the left and right ventricle). Two major papillary muscles connect the wall to the atrioventricular valve. (VanMieghen, 2010) The anterior papillary muscle is larger than the posterior. Occasionally a third papillary muscle is present. (Netter, 2010)

The outflow portion leads to the aorta through the aortic valve. The aortic valve, as the other semilunar valve, differs from the atrioventricular valves. There is not a defined annulus to support the valve. The first portion of the vessel expands to form three pouches, the sinus of Valsalva which are very obvious in the aorta. The wall of the vessel in this region is thinner than the aorta. The valvular leaflets are smooth and thin with a small fibrous nodule (nodulus Arantii) at the center of the free edge. Parallel to the free edges, a small area (lunula) of fine striations is evident. (Netter, 2010)

\subsection{Aorta and pulmonary artery}

The aortic semilunar valve is composed of three symmetric, semilunar-shaped cusps containing a recess called sinus of Valsalva. The junction of the sinuses and the aorta is called the sinotubular ridge since it makes a circular ridge. (Netter, 2010) When open, the valve forms a U-shape. The cusps are named based on the direction: the left and right (face the pulmonary valve), and the posterior. (Snell, 2008) The left and right have ostium on the inner surface opening into the left and right coronary arteries. The ostia are located below the sinotubular junction with the ostium of the left coronary; mildly superior and posterior to the right coronary ostium.The skeleton provides support to the structure. There is a small thickening on the center of the free edge of each cusp, the nodulus of Aramtius or Morgagni. The function of this nodule is to ensure complete closure. (Netter, 2010) From the nodule a line follows the free edge of the cusp, this line is called linea alba. Because of the increase aortic pressure, the linea alba, also present in the pulmonary cusps, is thicker and more pronounced. The plane of the aortic valve is mildly tilted.

The pulmonary valve resembles the structure of the aortic valve with the three symmetric, semilunar-shaped cusps. The cusps are attached to the right ventricular infundibulum and the pulmonary trunk. (Netter, 2010)

\subsection{Coronary blood flow}

Variations to the described anatomy are common. (Snell, 2008) The right coronary artery emerges from the right anterior sinus of Valsalva and runs in the right atrioventricular sulcus. Along this path the right coronary artery gives off two branches: the conus arteriosus branch and the right atrial branches. The conus artery and the communicating arteries in the interventricular septum serve as an important collateral blood supply to the left ventricle, anterior regions and anterior two-thirds of the interventricular septum. The right atrial branch gives the SA nodal artery (50-73\% of hearts), (Anderson, 1998; Iaizzo, 2005; Cohn, 2008) which runs along the anterior right atrium to the superior vena cava, encircling the vessels before reaching the SA node. As the right coronary artery reaches the AV groove, it gives several branches to the right atrium and ventricle, including the right marginal 
branch, which supplies the right margin of the heart. (Snell, 2008; Netter, 2010) The right coronary artery follows the sulcus posteriorly, curves until it reaches the crux cordis). It branches downward to form the posterior interventricular (descending) artery in the posterior interventricular sulcus. It supplies the posterior free wall of the right ventricle and in $85-90 \%$ branches into smaller arteries (posterior septal arteries) to supply the distal onethird of the interventricular septum. (Iaizzo, 2005) The atrioventricular nodal artery passes anteriorly to the base of the atrial septum and supplies the AV node (50-60\% of hearts), the proximal part of the bundles of His, and parts of the posterior interventricular septum. (Snell, 2008; Netter, 2010) The Kugel's artery originates from either the left or the right coronary and runs anterior to posterior toward the atrial septum, providing collateral connection from the anterior arteries to the AV node and the posterior arteries. (Saremi, 2008; Cohn, 2008)

The left coronary artery originates from the left sinus of Valsalva and emerges from the aorta between the pulmonary trunk and the left atrial appendage. Under the appendage the artery divides into the anterior interventricular (left anterior descending artery) and the left circumflex artery. The anterior interventricular (descending) artery follows the anterior interventricular sulcus, curves around the apex and anatomose with the posterior descending. It branches to give the anterior septal perforating arteries, which enter the septal myocardium and supply the anterior two-thirds of the interventricular septum. The first perforator reaches the AV conduction system, the second or third perforator is the longest of the septal arteries and the main septal artery. This artery reaches the middle portion of the interventricular septum and sends branches to the moderator band. The branches called the diagonal arteries, originating from the anterior descending artery, reach the anterior free wall of the left ventricle. These arteries are named in order of appearance (first diagonal, second diagonal etc). The anterior interventricular artery also supplies the right and left ventricular free walls. One branch meets the artery from the right coronary artery at the level of the conus artery to form the circle of Vieussens. (Snell, 2008; Netter, 2010) The circumflex branch of the left coronary artery runs in the left atrioventricular sulcus and supplies most of the left atrium, the posterior and lateral free walls of the left ventricle and the anterior papillary muscle of the mitral valve. It divides into several branches to supply the left ventricle. The terminal branch is the largest. It continues through the AV sulcus to supply the posterior wall of the left ventricle and the posterior papillary muscle of the mitral valve. The circumflex artery supplies the SA node in 40-50 \% of cases. (Iaizzo, 2005) In 30-60 $\%$, it is between the anterior and the circumflex artery, there are diagonal or intermediate arteries which extend toward the apex. (Iaizzo, 2005) In approximately $15 \%$ of patients the posterior descending artery also arises from the circumflex, while in $85 \%$ from the right coronary artery. Other variations to the normal pattern for both the left and the right coronary circulation are common in humans. (Snell, 2008; Netter, 2010)

The venous circulation is divided into three systems: 1 . the cardiac venous tributaries forming the coronary sinus, 2 . the anterior cardiac veins (anterior right ventricular), and 3. the smallest cardiac (Thebesian) venous system. The satellite venous system, formed by the great, middle and posterior (small) cardiac veins, converge to form the coronary sinus and drain $49 \%$ of myocardial blood. (Iaizzo, 2005, Snell, 2008) The anterior interventricular vein runs along the anterior interventricular sulcus with the anterior interventricular artery. Near the bifurcation of the left coronary artery, it turns and becomes the great cardiac vein. The great vein is formed by small tributaries from the left and right ventricle, and the anterior 
portion of the interventricular septum, the left atrium and the left ventricle. It also receives the large marginal vein which is parallel to the left marginal artery. The point of the great coronary vein becoming the coronary sinus is identified by the valve of Vieussens (a typical venous valve to prevent backflow), the space between the entry points of the oblique vein of the left atrium (vein of Marshall), and the posterior vein of the left ventricle. The oblique vein of Marshall runs superior to inferior along the posterior side of the left atrium, providing venous drainage of the area. It drains into the coronary sinus next to the great vein. The posterior vein ascends to the coronary sinus from the inferior portion of the left ventricle and provides drainage of the area. The coronary sinus also receives the middle vein. The veins, draining the posterior left and right ventricle and the interventricular septum, form the middle cardiac vein. This vein runs on the posterior interventricular sulcus and it enters the coronary sinus just before the right atrium. The small cardiac vein originates from the antero-lateral right ventricular wall and follows a path parallel to the marginal branch of the right coronary artery until it reaches the right atrioventricular sulcus. It turns and enters the coronary sinus with the middle cardiac vein. The small cardiac vein may be absent in $60 \%$ of the cases. In $50 \%$ of the cases it enters the right atrium directly. (Iaizzo, 2005) The anterior cardiac veins drain $24 \%$ of myocardial blood and form a separate circuit which does not drain into the coronary sinus. (Iaizzo, 2005) They drain into the anterior right ventricular wall and travel superiorly to cross the right AV sulcus to enter the right atrium directly. When present, the right marginal vein follows the right marginal artery and enters the right atrium. It is considered part of the anterior veins. The third system is composed of small intramural intramyocardial veins called Thebesian veins draining $17 \%$ of myocardial blood. (Iaizzo, 2005) These very small vessels don't have valves. They drain within the cardiac chambers via the Thebesian ostia in both the atria and the ventricles, but most commonly into the atrial and ventricular septa. They are more prevalent on the right side. (Netter, 2010)

\section{Anatomy of the conduction system}

The cardiac impulse arises in the sinoatrial (SA) node, located near the entrance of the superior vena cava. Known as the cardiac pacemaker, it generates the fastest rate of impulse. The impulse spreads to the interatrial and internodal conduction pathways to reach the atrioventricular (AV) node. The conduction travels to the bundle of His and then divides into left and right branches. Each bundle branch terminates in a network of fibers called the Purkinje fibers, whose stimulus generates ventricular contraction.

Under normal physiologic conditions the dominant pacemaker is the SA node, which in the adult fires at rate of 60 to 100 beat per minute (bpm). The overdrive suppression impedes other cells capable of spontaneous depolarization to become the dominant pacemaker. The cells located in the AV node and the Purkinje cells have a normal physiologic rate lower than the SA mode ranging from 25 to $55 \mathrm{bpm}$. (Iaizzo, 2005) In pathologic conditions the myocardial tissue itself can also exhibit self excitability generating ectopic beats. The parasympathetic system dominates at rest and slows the sinoatrial rate.

\subsection{Sinus node}

The SA node is located on the roof of the right atrium at the junction of the right atrial appendage, the superior vena cava, and the sulcus terminalis. In the adult it is $1 \mathrm{~mm}$ below 
the epicardium, 10-20 mm long and $5 \mathrm{~mm}$ thick. The P cells within the node are capable of spontaneous depolarization and are therefore responsible for generating the normal cardiac rhythm. (James, 2001) Importantly, the frequency of this depolarization is modulated by several factors both sympathetic and parasympathetic efferent innervation being the most important.

\subsection{Interatrial conduction}

After initial sinoatrial nodal excitation, depolarization spreads throughout the atria. The mechanism of impulse conduction through the atria is controversial. (Anderson, 1998; James, 2001) Excitation spreads through the myocardial atrial tissue cell-to-cell using specialized connections between the cells called gap junctions. Additional specialized conduction cells may be organized in pathways and constitute the internodal and interatrial pathways. It is believed, however, that there are three anatomic conduction pathways originating within the nodal tissue of the SA node or in the proximity of the node. The internodal tracts include the anterior internodal tract which extends from the anterior regions of the sinus node, travels on the roof of the right atrial septum and bifurcates into the Bachman's bundle directed to the left atrium and a second tract descending to the AV node, along the anterior part of the interatrial septum. The middle internodal tract (or Wenckebach's pathway) descends within the septum anteriorly to the fossa ovalis and reaches the AV node. It is the most inconsistent and not well developed. The third tract is called posterior internodal tract (Thorel's), which passes along the crista terminalis through the Eustachian valve, posteriorly to the coronary sinus. (James, 2001)

\subsection{Atrioventricular node and His bundle}

The AV node, also called node of Tawara, is located on the floor of the right atrium included within the triangle of Koch. $(\mathrm{Ho}, 2006)$ The proximal AV bundle continues into the portions of the AV node: transitional zone and compact node. It continues with the penetrating distal AV bundle (His bundle). (Anderson, 1998) The excitation from the SA travels through the AV node tissue following two functional and anatomical pathways, the slow and the fast pathways. The slow pathway crosses the isthmus between the coronary sinus and the tricuspid annulus and has slower conduction velocity but a shorter effective refractory period. (Anderson, 1998) The fast pathway is located superiorly and the fibers enter the node transversally in the distal portion of the compact node. It has a shorter conduction but a longer effective refractory period. Normal conduction occurs along the fast pathways, however, premature beats and higher rates find the fast pathway during the refractory period and conduct along the slow pathway. This system is a protective mechanism. In normal conditions the AV node-His bundle represents the only communication between the atria and ventricle. However, direct connections to the ventricular myocardium through the fibrous skeleton have been found. They constitute accessory pathways. The Mahaim fibers connect the penetrating portion of the distal bundle and the AV node to the ventricular myocardium. An additional aberrant pathway is the bundle of Kent. (Iaizzo, 2005)

\subsection{Bundle branches}

The AV bundle from the AV node penetrates the central fibrous body and passes through the membranous septum as a common bundle and splits into left and right bundle branches at the apex of the muscular septum. The left bundle branch splits into fascicles as it travels 
into the left subendocardic portion of the interventricular septum. Midway to the apex of the left ventricle, the left bundle splits into two major divisions, the anterior and posterior branches or fascicles. The right bundle continues inferiorly as a continuation of the bundle of His in the subendocardic portion of the interventricular septum.(James, 2001; Snell, 2008)

\subsection{Purkinje fibers}

The Purkinje fibers constitute a network of conduction specialized fibers arising from both left and the right bundle branches. They are characterized by rapid conduction. The fibers extend within the myocardium and the trabeculation of the right and left ventricle. One of the most common conduction pathways is the moderator band, which contains Purkinje fibers from the right bundle branch. (Snell, 2008)

\section{References}

Anderson PAW. (2000). Developmental cardiac physiology and myocardial function. In: Pediatric cardiovascular medicine, Moller JH, Hoffman JIE, eds., pp. 35-57,. Churchill Livingstone, New York

Anderson RH, Cook AC. (2007) The structure and components of the atrial chambers. Europace, 9 Suppl 6:vi3-9

Anderson, RH, Ho, SY.(1998) The architecture of the sinus node, the atrioventricular conduction axis, and the internodal atrial myocardium, J Cardiovasc Electrophysiol.. 9(11), pp.1233-1248

Angelini, P (1995) Embryology and congenital heart disease. Tex Heart Inst J., 22(1): 1-12

Aquino, SL • Gregory R. Duncan, MS • L. Anne Hayman, MD (2001). Nerves of the Thorax: Atlas of Normal and Pathologic Findings, RadioGraphics, 21, pp.1275-1281

Bolling SF (2006) Functional anatomy of the mitral valve, In: Atlas of mitral valve surgery, Savage EB, Bolling SF eds pp.7-10, Lippincott Williams \& Wilkins, Philadelphia, US

Colvin EV (1998) Cardiac embryology. In: The Science and Practice of Pediatric Cardiology Garson AJr (Eds.), (2nd), pp 91-126, Williams \& Wilkins, Baltimore

DeHaan RL. (1963). Organization of the cardiogenic plate in the early chick embryo. Acta Embryol Morphol Exp, 6, pp. 26-38

Friedman AH, Fahey JT. (1993) The transition from fetal to neonatal circulation: normal responses and implications for infants with heart disease. Semin Perinatol. 17(2):106121

Harvey RP, Rosenthal N. (1999) Heart development. (1st). Academic Press, San Diego

Hildreth V, Webb S, Bradshaw L, Brown NA, Anderson RH,Henderson DJ. (2008). Cells migrating from the neural crest contribute to the innervation of the venous pole of the heart. J Anat, 212, pp. 1-11

Ho SY, Anderson RH, Sánchez-Quintana D. (2002) Atrial structure and fibres: morphologic bases of atrial conduction. Cardiovasc Res., 54(2), pp. 325-336

Ho SY, Sánchez-Quintana D. (2009) The importance of atrial structure and fibers. Clin Anat., 22(1), pp. 52-63

Iaizzo PA (Ed) (2009). Handbook of Cardiac Anatomy, Physiology, and Device (2nd) ISBN: 9781603273718, NY

James TN.(2001) The internodal pathways of the human heart. Prog Cardiovasc Dis., 43(6), pp. $495-535$ 
Jason H. Rogers, MD; Steven F. Bolling, MD The Tricuspid Valve Current Perspective and Evolving Management of Tricuspid Regurgitation. Circulation. 2009;119:2718-2725

Kelly RG, Buckingham ME. (2002). The anterior heart-forming field: Voyage to the arterial pole of the heart. Trends Genet, 18, pp. 210-216

Kirby ML. (2002). Molecular embryogenesis of the heart. Pediatr Dev Pathol, 23, pp. 537-544

Komiyama M, Ito K, Shimada Y. (1996) Origin and development of epicardium in the mouse embryo. Anat Embryol, 176, pp. 183-189

Larsen WJ (1997b) Human Embryology, (2nd), Churchill Livingstone, New York

McCarthy KP, Ring L, Rana BS. Anatomy of the mitral valve: understanding the mitral valve complex in mitral regurgitation.Eur J Echocardiogr. 2010 Dec;11(10):i3-9.

Michael R. Mill, Benson R. Wilcox, and Robert H. Anderson (2008) Surgical Anatomy of the Heart, In: Cardiac surgery in the adult (3rd), Cohn SH ed, pp.29-50, ISBN 0071391290, McGrawHilld Education

Mikawa T, Gourdie RG. (1996) Pericardial mesoderm generates a population of coronary smooth muscle cells migrating into the heart along with ingrowth of the epicardial organ. Dev Biol, 173, pp 221-232

Netter FH, (2010) Netter FH, Dalley AF, eds. Atlas of human anatomy. (5 $\left.{ }^{\text {th }}\right)$. Navartis, East Hanover, NJ

Pensky B (1982) Review of Medical Embryology., McMillan, New York

Poelmann RE, Gittenberger-de Groot AC. (1999). A sub-population of apoptosis prone cardiac neural crest cells targets the venous pole: Multiple functions in heart development?, Dev Biol, 207, pp. 271-286

R. Abdulla, G. A. Blew, M.J., Holterman MJ (2004). Cardiovascular Embryology. Pediatr Cardiol 25, pp. 191-200

Rogers JH, Bolling SF.The tricuspid valve: current perspective and evolving management of tricuspid regurgitation. Circulation. 2009 May 26;119(20):2718-25.

Saremi F, Abolhoda A, Ashikyan O, Milliken JC, Narula J, Gurudevan SV, Kaushal K, Raney A (2008) Arterial supply to sinuatrial and atrioventricular nodes: imaging with multidetector CT. Radiology. 246(1), pp. 99-107

Sherman LS, Potter SS, Scott WJ, eds. (2001) Human embryology. (3rd). Churchill Livingstone, New York

Snell, RS.(2008) The thorax: part II-The thoracic cavity, In Clinical anatomy by regions, pp. 77130, ISBN 0781764041, Lippincott Williams \& Wilkins, Philadelphia, US

Srivastava D, Olson EN. (2000). A genetic blueprint for cardiac development. Nature, 407, pp. 221-226

Steding G, Seidl W (1984) Cardiac septation in normal development. In: Nora JJ, Talao A(Eds.), Congenital Heart Disease: Causes and Processes. pp 481-500, Futura, New York,

Van Mieghem NM, Piazza N, Anderson RH, Tzikas A, Nieman K, De Laat LE, McGhie JS, Geleijnse ML, Feldman T, Serruys PW, de Jaegere PP. (2010) Anatomy of the mitral valvular complex and its implications for transcatheter interventions for mitral regurgitation. J Am Coll Cardiol. 17;56(8), pp 617-626

Van Mierop LHS (1979) Morphological development of the heart. In: Berne RM (Eds.), Handbook of Physiology, the Cardiovascular System. pp 1-28American Physiology Society, Bethesda, MD 
Waldo K, Miyagawa-Tomita S, Kumiski D, Kirby ML. (1998) Cardiac neural crest cells provide new insight into septation of the cardiac outflow tract: Aortic sac to ventricular septal closure. Dev Biol, 196, pp. 129-144

Wessels, M.W.M. Markman, J.L.M. Vermeulen, R.H. Anderson, A.F.M. Moorman, W.H. Lamers (1996) The Development of the Atrioventricular Junction in the Human Heart Circulation Research. 78:110-117 


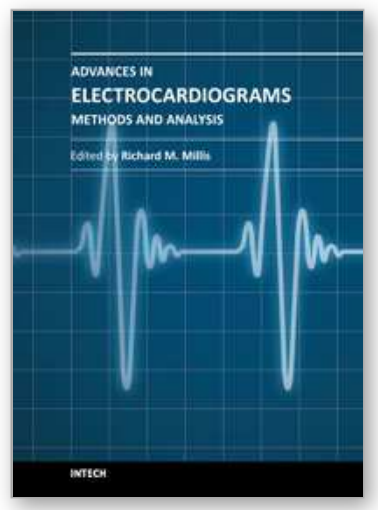

\author{
Advances in Electrocardiograms - Methods and Analysis \\ Edited by PhD. Richard Millis
}

ISBN 978-953-307-923-3

Hard cover, 390 pages

Publisher InTech

Published online 25, January, 2012

Published in print edition January, 2012

Electrocardiograms are one of the most widely used methods for evaluating the structure-function relationships of the heart in health and disease. This book is the first of two volumes which reviews recent advancements in electrocardiography. This volume lays the groundwork for understanding the technical aspects of these advancements. The five sections of this volume, Cardiac Anatomy, ECG Technique, ECG Features, Heart Rate Variability and ECG Data Management, provide comprehensive reviews of advancements in the technical and analytical methods for interpreting and evaluating electrocardiograms. This volume is complemented with anatomical diagrams, electrocardiogram recordings, flow diagrams and algorithms which demonstrate the most modern principles of electrocardiography. The chapters which form this volume describe how the technical impediments inherent to instrument-patient interfacing, recording and interpreting variations in electrocardiogram time intervals and morphologies, as well as electrocardiogram data sharing have been effectively overcome. The advent of novel detection, filtering and testing devices are described. Foremost, among these devices are innovative algorithms for automating the evaluation of electrocardiograms.

\title{
How to reference
}

In order to correctly reference this scholarly work, feel free to copy and paste the following:

Augusta Pelosi and Jack Rubinstein (2012). Cardiac Anatomy, Advances in Electrocardiograms - Methods and Analysis, PhD. Richard Millis (Ed.), ISBN: 978-953-307-923-3, InTech, Available from:

http://www.intechopen.com/books/advances-in-electrocardiograms-methods-and-analysis/cardiac-anatomy

\section{INTECH}

open science | open minds

\section{InTech Europe}

University Campus STeP Ri

Slavka Krautzeka 83/A

51000 Rijeka, Croatia

Phone: +385 (51) 770447

Fax: +385 (51) 686166

www.intechopen.com

\section{InTech China}

Unit 405, Office Block, Hotel Equatorial Shanghai

No.65, Yan An Road (West), Shanghai, 200040, China 中国上海市延安西路65号上海国际贵都大饭店办公楼 405 单元

Phone: $+86-21-62489820$

Fax: $+86-21-62489821$ 
(C) 2012 The Author(s). Licensee IntechOpen. This is an open access article distributed under the terms of the Creative Commons Attribution 3.0 License, which permits unrestricted use, distribution, and reproduction in any medium, provided the original work is properly cited. 